\title{
La autoevaluación de los estudiantes un proceso por resignificar y reconstruir en la educación física escolar
}

\author{
The student self-assessment process by resignificar and rebuild in school \\ physical education
}

\section{Processo de auto-avaliação do aluno por resignificar e reconstruir na educação física escolar}

\section{Luz Stella García Carrillo}

Doctora en ciencias de la educación área pedagogía Docente de planta programa de educación física, deporte y recreación Grupo de investigación Devenir Evaluativo

Universidad del Tolima

lsgarcia@ut.edu.co

\section{Resumen}

Este artículo presenta algunos planteamientos para la reflexión y debate sobre la evaluación y específicamente la autoevaluación de los estudiantes en la Educación Física escolar, dado que en los últimos años hay un marcado interés por la evaluación en sus diferentes ámbitos y niveles educativos porque se reconoce la necesidad de estudiar la evaluación educativa sus avances teóricos e investigativos para orientar y desarrollar prácticas evaluativas que permitan aprender y mejorar.

La autoevaluación de los estudiantes en la Educación Física escolar generalmente es una práctica aún ausente o tergiversada, ya que se confunde la autoevaluación con asignar o negociar la calificación con el profesor lo que distorsiona sus fundamentos, características y usos. Se desconoce su importancia y aporte para la formación.

En el texto se destaca la autoevaluación de los estudiantes como un proceso de alto valor pedagógico y se esbozan algunos conceptos, características y recomendaciones para su implementación en la Educación Física escolar que permitan su resignificación.

Palabras claves: autoevaluación de los estudiantes, participación, auto calificación, proceso formativo, Educación Física escolar. 


\section{Summary}

This paper presents some approaches for reflection and debate on the evaluation and specifically self-assessment of students in school physical education, because in recent years there is a strong interest in evaluation in different areas and educational levels because it recognizes the need to study theoretical educational evaluation and research advances to guide and develop assessment practices to learn and improve.

Self-evaluation of students in physical education at school is usually absent or distorted even practice since the self-assessment score assign or negotiate with Professor distorting its foundations, characteristics and uses confused. Its importance and contribution to the formation is unknown.

In the text the student self-assessment stands out as a highly educational process and some concepts, features and recommendations for implementation in physical education in schools that allow their resignification outlined.

Keywords: self-assessment of students, participation, self qualification, training process, school physical education.

\section{Resumo}

Este artigo apresenta algumas abordagens para reflexão e discussão sobre a avaliação e, especificamente, a auto-avaliação dos alunos em educação física escolar, já que nos últimos anos há um interesse acentuado na avaliação em diferentes áreas e níveis de ensino, porque reconhece o precisa estudar avanços de avaliação educacional e de pesquisa teórica para orientar e desenvolver práticas de avaliação para aprender e melhorar.

A auto-avaliação dos alunos em educação física escolar é geralmente ausente ou deturpado até mesmo praticar desde a qualificação de atribuição de auto-avaliação ou negociar com Professor distorcendo suas fundações, características e usa confuso. Sua importância e contribuição para a formação é desconhecida.

No texto, o aluno auto-avaliação destaca-se como um processo altamente educativo e alguns conceitos, características e recomendações para a implementação na educação física nas escolas que permitem a sua ressignificação delineado.

Palavras-chave: auto-avaliação dos alunos, participação, auto de qualificação, processo de formação, de educação física escolar.

\section{Introducción}

El termino autoevaluación posee varias acepciones, en el presente texto me referiré a la autoevaluación de los estudiantes como un proceso pedagógico y formativo en la Educación Física escolar y no a la autoevaluación para la certificación y acreditación de programas e instituciones educativas. La evaluación educativa y especialmente la evaluación de los estudiantes es un proceso con 
componentes políticos, pedagógicos y éticos que reflejan los intereses, las tensiones e incoherencias del sistema educativo.

Evaluar para muchos es sinónimo de medir y calificar, se aclara que evaluar, implica: por un lado calificar para dar un resultado expresado en una nota dado que la evaluación tiene como una de sus funciones reconocidas socialmente el certificar el aprendizaje. Por otro lado, y al mismo tiempo, pero con finalidades diferentes, evaluar es valorar el proceso para aprender y formar. Es decir, calificar y evaluar son dos actividades complementarias e igualmente determinantes. La "medición y evaluación son sinónimos inseparables y actividades intercambiables (Díaz, 2005, p.21).

No se puede hablar en la evaluación educativa "de un solo enfoque y modelo de enseñanza y evaluación, sino un amplio abanico de aproximaciones modélicas y metodológicas que el profesorado debe manejar y gestionar, en función de su contexto pedagógico particular, disciplina, nivel, tipo de curso, características del estudiante" (Escudero, 2010, p.5). La evaluación "se mueve en un amplio rango de posiciones teóricas que sustentan $y$ dan sentido a multiplicidad de prácticas evaluativas que reflejan algunos intereses, tensiones e incoherencias del sistema educativo (García Carrillo, 2012).

Debe quedar claro que el proceso formativo del estudiante transciende el medir para entregar unos resultados expresados en una nota exclusivamente; afortunadamente "la evaluación no solo mide lo aprendido, ni siquiera valora lo enseñado, también enseña por sí misma" (Murillo e Hidalgo, 2015, p. 5). En este sentido, se observa un lento pero significativo avance en el reconocimiento y comprensión de la evaluación como un proceso multidimensional.

La autoevaluación es una de las prácticas más desconocida y tergiversada en la Educación Física escolar y en las demás áreas. La autoevaluación es una posibilidad desaprovechada y poco valorada al evaluar al estudiante; "se trata éste de un aspecto olvidado en la práctica docente, pero si ayuda a que el alumno sea más consciente de su propia realidad y que el profesor la conozca mejor" (Blázquez, 2008, p. 56).

En este orden de cosas, hay mucho por hacer, sí se quiere transformar el sentido y significado de la evaluación de los estudiantes desde una perspectiva formativa; es importante conocer, reflexionar e incorporar el concepto, principios, características, procesos y procedimientos de autoevaluación de los estudiantes, pero aun es más importante comprender el papel que desempeña en el aprendizaje. Por esta y otras razones la autoevaluación está en proceso de resignificación y reconstrucción.

Una preocupación urgente en la Educación Física escolar, es superar las prácticas evaluativas tradicionales $\mathrm{y}$ anquilosadas en posturas exclusivamente sicométricas donde se evalúa a través de los test de condición física y motriz y pruebas motrices de habilidad o de valoración técnica que son necesarias pero no son la única y exclusiva forma de evaluar. Porque el problema de fondo, es que posiblemente se sigue enseñando lo mismo de siempre y de la misma manera y por consiguiente y en correspondencia evaluando de igual forma, aunque hay que reconocer que se están proponiendo e 
implementando lentamente otras formas de ver y aplicar la evaluación de los estudiantes en la Educación Física escolar en el marco de una enseñanza y aprendizaje autónomo, significativo y cooperativo.

En Colombia y en otros países: Camacho (2015), Díaz Lucea (2005), Fernández Balboa (2005), López (2004), Fraile (2004), Blázquez, (2008-2016), Guio (2012), García Carrillo (2012) entre otros han estudiado e investigado sobre el tema con valiosos aportes.

\section{Ausencia, confusión y tergiversación en la autoevaluación}

La aplicación de la evaluación en el aula en algunas ocasiones, dista de estar estructurada y orientada a la formación del estudiante y a la mejora institucional porque, existe confusión por "la mezcla de funciones que se le asignan a la evaluación educativa. Es evidente que desempeña muchas y muy variadas: formación, selección, certificación, ejercicio de autoridad, mejora de la práctica docente; funciones relacionadas con la motivación y la orientación; funciones administrativas, académicas de promoción o de recuperación; de información y de retroalimentación, de control," (Álvarez Méndez, 2001, p. 24).

Son muchos los factores que subyacen y afectan la comprensión de la evaluación educativa como los profundos cambios e intereses políticos, sociales y culturales, así como la economía de mercado, los procesos de globalización del capital y el desarrollo de la sociedad de la información y de la comunicación, que traen consigo condiciones y problemas que se reflejan en el sector educativo.
No se puede olvidar otro factor determinante, como es la perspectiva o tendencia de la Educación Física, que responde a la pregunta ¿cuáles son las orientaciones epistemológicas, antropológicas, pedagógicas, curriculares y didácticas que fundamentan el área?, una tendencia de la Educación Física escolar se configura y corresponde a unas prácticas con fundamentos epistemológicos, políticos, sociales y pedagógicos; por ejemplo: "el hecho de asumir la Educación Física como una práctica de ejercicio físico más que como acción educativa, orienta las tendencias de evaluación hacia una limitada noción técnica, donde se reconoce como proceso básico la recolección de información y la emisión de juicios valorativos" (Guio, 2012, p.863) que desfigura su importancia y función formativa.

Se puede afirmar que el concepto de autoevaluación fue acuñado hace algún tiempo y ha evolucionado pero aun es una práctica asistemática y ausente en las aulas; por otro lado, esta tergiversada; de acuerdo a la Real Academia de la Lengua tergiversar "es dar una interpretación forzada o errónea a palabras o acontecimientos, ya que cuando se aplica se desconoce $y$ no es clara su fundamentación teórica.

Cambiar la cultura de la autoevaluación no es responsabilidad y un propósito exclusivo del profesor, ya que depende de muchos factores $\mathrm{y}$ condiciones. $\mathrm{El}$ profesor juega un papel decisivo en la puesta en acción de la autoevaluación al proponer y aplicar un sentido a la evaluación y una variada gama de técnicas e instrumentos de autoevaluación que enriquecen el proceso formativo. 
Los postulados, situaciones, condiciones y propuestas frente a la autoevaluación de los estudiantes son válidos para todas las áreas fundamentales y optativas del plan de estudios en la Educación Básica y Media, no se puede considerar un hecho o problema particular y exclusivo de la Educación Física escolar.

\section{La autoevaluación un proceso ausente o tergiversado}

El desconocimiento y la poca claridad sobre la autoevaluación es uno de los problemas de fondo. Como concepto y práctica generalmente se confunde con la auto calificación. En todos los niveles educativos; se considera que autoevaluar es simplemente que el estudiante se coloque o negocie con el profesor una nota sin una reflexión crítica sistemática y continua y sin tener en cuenta sus avances, limitaciones y errores en el aprendizaje y el desarrollo del estudiante.

López (2004) y López, Barba, González (2005) en una de sus publicaciones sobre la evaluación en la Educación Física escolar, plantean el término "calificación dialogada" la calificación dialogada es una consecuencia lógica y coherente de un proceso de evaluación compartida, es importante tener claro que son dos procesos diferentes y, que a pesar de que en algún caso puedan estar relacionados" (López, Barba, González, 2006, p.3) es una aproximación que no se equipara con la autoevaluación porque se corre el peligro de quedarnos solo en ello.

Comúnmente, los estudiantes la identifican como "nota apreciativa", supuestamente es un reconocimiento o "regalo" del profesor por el trabajo desarrollado. Se destaca más el resultado y prima el interés de obtener una buena nota que permita pasar o subir la calificación en una participación mecánica y procedimental que no provoca reflexión, aprendizaje, formación en las diferentes dimensiones del desarrollo humano. Desde y a partir de la evaluación y la autoevaluación se realizan juicios frente al profesor, ajustes y se rinde cuentas.

En el mismo sentido, Díaz (2005), afirma:

...el alumno no participa o participa poco: es una práctica poco frecuente dejar que el alumno participe en su evaluación y, cuando se hace, nos limitamos a dejar que anote o a pedirle la opinión sobre que nota cree que le tiene que poner. Son muy pocos los casos en que los alumnos puedan decidir plenamente en su evaluación. Es necesario plantear la autoevaluación y articular formas para conseguirlo (Díaz, 2005, p.55).

Por ello, es pertinente reconocer y orientar la autoevaluación de manera formativa, integral y motivadora, procurando que cada estudiante identifique los aspectos en los que ha avanzado o los que presenta limitaciones y dificultades, para autorregular sus procesos a través de actividades de mejora en la consecución de los objetivos, metas y competencias previstas por la Institución.

\section{Desconocida por la comunidad educativa}

Para dejar de ser una "desconocida" es necesario tener la intensión de convertirla en una práctica permanente e incorporarla en la cultura evaluativa, ya que las actividades vinculadas a la autoevaluación, actualmente no tienen mayor importancia. Nuestra cultura 
evaluativa en la mayoría de los casos, no enseña a autoevaluarnos.

Según Gómez (2012) uno de los mayores errores que se pueden cometer:

...es enfatizar los productos sobre los procesos, reducir los productos a respuestas idénticas para todos y desvincular los productos de los procesos y los procesos de los contextos. La evaluación educativa no puede obviar la multiplicidad de variables que están interviniendo ni la complejidad de las interacciones que se establecen entre ellas al formar los sistemas de comprensión, toma de decisiones y actuación (Pérez, 2012, p. 240).

La evaluación, en el sistema educativo colombiano, generalmente no ha ocupado un lugar relevante, ha sido autoritaria, definida en forma vertical por el Ministerio de Educación Nacional en sus documentos y normas donde propone algunas renovadas e interesantes posturas sobre la evaluación formativa; desafortunadamente muchos profesores aun no conocen estas propuestas y mucho menos las han apropiado e incorporado a sus prácticas cotidianas para enseñar y aprender. Murillo e Hidalgo (2015) reiteran que:

Es necesario se promueva activamente la implicación y el compromiso activo de toda la comunidad en dicha evaluación, especialmente de los directamente afectados, empoderar a los estudiantes en su propia evaluación y la de sus compañeros se traduce en un sentimiento de responsabilidad en el propio aprendizaje, lo que sin dudas tiene fuertes implicaciones en su participación activa en la sociedad. Este empoderamiento, también ha de traducirse en actividades de coevaluación, autoevaluación, así como el consenso con los docentes de contenidos, estrategias, momentos y criterios de evaluación (p.6)

Las políticas y programas estatales muestran un esfuerzo técnico y financiero en la formación permanente de los profesores orientada a la mejora $y$ desarrollo profesional con programas de acompañamiento en la institución y en el aula para actualizar y profundizar en aspectos curriculares, didácticos y evaluativos, especialmente en la evaluación de los estudiantes de algunas áreas. Por otro lado, simultáneamente el MEN implementa la evaluación masiva y estandarizada nacional que no incluye la Educación Física pero afecta la valoración, comprensión y desarrollo de la evaluación en la Educación Física escolar.

\section{Preparación débil e insuficiente de los profesores}

Los profesores se interesan por la evaluación pero algunos presentan problemas de conceptualización y contextualización. " así como posiciones de cierta ingenuidad, que piensan que los problemas de enseñanza son básicamente problemas de evaluación, y que estos son básicamente problemas de naturaleza técnica, ligados a los procedimientos de evaluación"(Escudero, 2010, p.4).

Prevalece una concepción reduccionista de la evaluación "no es una práctica habitual para la mayor parte del profesorado" (Castillo y Cabrerizo, 2009, p.221), en algunos casos se desconoce que la evaluación y la autoevaluación son una responsabilidad directamente compartida por cada estudiante $y$ el docente. 
Pero no es fácil, la solución transciende las palabras, para llevar a cabo las acciones orientadas a mejorar la evaluación, es urgente e inaplazable la formación de todos los actores de la comunidad educativa, no es solo pensar que para formar a los profesores basta con un evento, conferencia o taller para transformar el sentido, significado y práctica de la evaluación. Se requiere un sistema de formación, actualización y capacitación docente articulado y en función al trabajo en el aula.

\section{La finalidad e impacto de la autoevaluación de los estudiantes no es clara}

La autoevaluación de los estudiantes al estar ausente o tergiversada como un procedimiento sin sentido, poca promovida y con limitados desarrollos que no corresponden a sus finalidades y características formativas. Indudablemente la autoevaluación trae consigo mejoras en el aprendizaje y ante todo favorece la participación activa de los estudiantes ya que propicia la autoreflexión, la actitud crítica, el autogobierno, el sentido de responsabilidad, respeto y confianza.

Su implementación permite, reconocer y posicionar al estudiante como el principal actor del proceso educativo que participa activamente como agente determinador con mayor compromiso personal y académico para lograr mejores procesos y resultados.

Según Castillo y Cabrerizo, (2010) la autoevaluación sirve al alumno para:

- Reconocer sus avances, logros y dificultades.
- Analizar su actuación individual y grupal en el proceso educativo.

- Desarrollar una actitud crítica y reflexiva.

- Autorregular su proceso de aprendizaje.

Sirve al docente para:

- Disponer de mejores elementos de juicio que le permitan facilitar y reorientar el aprendizaje de sus alumnos.

- Valorar la actuación y conocer la situación de los alumnos.

- Valorar su propia actuación y adecuar los programas, modificando contenidos, metodologías, etc.

La autoevaluación puede generar o no, una nota o calificación. Para algunos autores y mi postura personal es que en lo posible no este atada a la nota. La autoevaluación puede emitirse de manera oral o escrita, la información obtenida es necesario se registre en las planillas o formatos diseñados para tal fin. Estos resultados de la autoevaluación pueden ser emitidos en forma cualitativa mediante escalas numéricas o porcentajes y enunciados.

En una radiografía de lo que sucede y continúa sucediendo en muchos casos, persiste el desconocimiento de la autoevaluación como proceso pedagógico y su implementación es muy baja en la Educación Física escolar.

\section{Referentes conceptuales de la autoevaluación}

En la autoevaluación de los estudiantes se pueden identificar dos vertientes teóricas 
de la autoevaluación. La autoevaluación como estrategia de (para el) aprendizaje y la autoevaluación como estrategia pedagógica. Estas dos vertientes son complementarias.

En la autoevaluación como estrategia de aprendizaje el alumno tiene un papel activo. La autoevaluación como un proceso instruccional que el profesor utiliza como recurso pedagógico. Como parte de la evaluación formativa. En la autoevaluación como estrategia pedagógica a través de la cual el profesor pide a sus alumnos que reflexionen sobre su trabajo, se sustenta en teorías sobre autorregulación.

En términos generales se puede decir que la autoevaluación es la evaluación realizada por la propia persona sobre sí misma, se identifica en la literatura inglesa como self-assessment. La autoevaluación, se fundamenta en el desarrollo de principios que favorecen la autonomía, la participación y crecimiento personal. El estudiante, al ser agente activo, de su propio proceso de evaluación, a partir de la autoreflexión identifica sus fortalezas y debilidades en el aprendizaje.

A continuación se presentan varios conceptos de autoevaluación:

En Román (2011) la autoevaluación como proceso permite la discusión y reflexión sobre lo hecho y lo logrado, con el propósito de reorientar actividades y estrategias.

Para Boud (1995) "La autoevaluación consiste en que los alumnos desarrollen las habilidades de aprendizaje. No es solo una técnica de evaluación entre otras" (p.15).
Para Santos Guerra (1993) "La autoevaluación es un proceso de auto crítica que genera unos hábitos enriquecedores de reflexión sobre la propia realidad.... No es aceptable la práctica del juego auto evaluador, en la cual el alumno hace reflexiones y análisis de su aprendizaje, pero no puede materializar el resultado en una parcela de las calificaciones" (p.41).

Castillo y Cabrerizo (2010) "La autoevaluación busca que cada estudiante participe en la valoración de sus actividades de aprendizaje, "es motivadora porque el alumno cuando se autoevalúa, no solamente está asumiendo un mayor protagonismo en su proceso de aprendizaje, sino que además, está desarrollando la capacidad de valorar su proceso educativo, lo que implica asumir también una mayor responsabilidad en sus actuaciones" (p. 188).

Para Panadero y Alonso - Tapia (2013) la autoevaluación consiste en: "la valoración cualitativa de la ejecución, esto es, proceso de aprendizaje y del producto final del mismo, valoración realzada a partir de unos criterios preestablecidos y modulada por los niveles de perfección que el alumno desee alcanzar" (Pandero, 2009, p. 78).

Para Camacho, Castillo y Monje (2015) "La autoevaluación es la valoración que cada individuo hace de su propio trabajo, refleja la autoestima y el concepto de trabajo que se maneja individualmente.

Revela datos importantes que solo conoce quien se autoevalúa y que frecuentemente son determinantes del comportamiento humano, su sentido de responsabilidad y permite considerar aspectos que solo 
quien se autoevalúa conoce a profundidad".

Según Lopez Pastor (2006) la autoevaluación favorece la reflexión, la actitud crítica y el sentido de responsabilidad. El estudiante se valora a sí mismo y sus aprendizajes son más significativos puesto que esta tiene carácter formativo y no deriva en nota alguna, su propósito es reflexionar sobre el desempeño y las actitudes de los estudiantes con el fin de optimizar el aprendizaje.

- Este proceso de autoevaluación debe estar encaminado de tal manera que sus resultados sean de utilidad para los estudiantes, el docente y el grupo con el fin de motivar el desarrollo del aprendizaje.

- Es importante que promueva la reflexión, la auto observación y el análisis de las situaciones que se han presentado en transcurrir del periodo.

Según García Carrillo (2016) “es el proceso de reflexión y de revisión eminentemente formativo que permite la participación activa del estudiante. Se fundamenta en la auto determinación y auto regulación al reconocer y valorar sus propios objetivos, procesos y aprendizajes. No es simplemente un formulismo para negociar una nota $o$ llenar un formato". Desde el punto de vista didáctico la evaluación es un recurso para realimentar que permite ajustar, corregir, reformular y mejorar.

\section{Recomendaciones para desarrollar la autoevaluación de los estudiantes}

Estas recomendaciones son un punto de partida; no hay una forma o formula única para realizarla. Pero ante todo "la evaluación para y como aprendizaje tiene que ser personal, retadora y relevante si queremos que implique al estudiante" (Pérez, 2012, p.240). La evaluación y autoevaluación de los estudiantes debe ser una práctica que responda a las condiciones, características y necesidades particulares de cada contexto educativo.

Al igual que las actividades de evaluación se recomienda que el profesor incluya la autoevaluación en su planeación curricular. El prever estas actividades con anticipación permite informar de manera clara y precisa al estudiante y definir los procesos y valores que se ponen en juego.

\section{Su aplicación debe ser progresiva}

Una de las investigaciones sobre la evaluación en la Educación Física de Guio (2012) nos da pistas sobre este aspecto en el país, "en general, los estudiantes tienen una actitud pasiva frente a la construcción de los criterios":

Los estudiantes son conscientes de la evaluación, se ajustan a las condiciones planteadas con actitud normal, acatan los criterios propuestos, generalmente no los discuten, son conscientes que si trabajan en la medida de sus posibilidades van a pasar y la asumen con actitud positiva (Guio, 2012, p. 25).

Cuando al iniciar el año escolar se discuten los acuerdos pedagógicos o contratos didácticos los estudiantes se preocupan mucho más por el número de 
evaluaciones y su valor en la nota final. Como bien lo explica Fernández - Balboa (2005).

... quizás la mayor lucha que tengo al proponer y practicas la autoevaluación es romper el previo condicionamiento de los estudiantes, los cuales durante sus años de escolaridad se han acostumbrado a que se les diga no solo lo que han de hacer, cómo deben hacerlo, y para cuándo deben hacerlo, sino que también esperan que el profesor "valore" todo ello con una nota sin darles muchas explicaciones (p. 148).

Lo ideal es evaluar y autoevaluar según las características individuales de los estudiantes, los ritmos de aprendizaje y el desarrollo motor entre otras; pero las condiciones de nuestras aulas e instalaciones y el elevado número de estudiantes, dificultan su desarrollo. Es allí donde tiene sentido una autoevaluación contextualizada, progresiva, comprensiva y transparente.

El profesor al fomentar la autoevaluación debería emplearla como estrategia instruccional cuyo objetivo final debería ser guiar a los alumnos para que aprendiesen a autoevaluarse, de modo que este aprendizaje repercutiese en su autorregulación (Panadero y Alonso Tapia, 2013, p. 555).

Castillo y Cabrerizo (2010) proponen algunos los criterios a tener en cuenta en los contenidos objeto de autoevaluación que para ser también válidos y adaptables a la Educación Física escolar deberán:

- Estar previstos de antemano.

- Haber sido previamente explicados.
- Ajustarse al resto de contenidos contemplados en la programación y estar en relación con ellos.

- Pertenecer a la materia a evaluar.

- Ser idóneos para el nivel del alumnado que se autoevalúe.

La autovaloración obtenida de la autoevaluación de los contenidos debería ser complementaria y contrastada con la valoración que aporten otros instrumentos y técnicas de evaluación (p. 207).

\section{La participación}

En la óptica de una evaluación formativa se ratifica la participación como un eje, "la autoevaluación hace referencia a la participación de los aprendices en la realización de juicios acerca de su propio aprendizaje, particularmente de sus logros y de los resultados de su aprendizaje" (Boud y Falchikov, 1989) citado por Dorchy, Segers y Dierick (2002).

El dialogo pedagógico cooperativo y feedback, dos elementos imprescindible. El feedback persigue un objetivo global de llevar o conducir a los estudiantes a supervisar su propia actuación. Es aconsejable que para que el feedback sea eficiente es necesario se produzca de manera inmediata y acompañando el aprendizaje.

Se insiste en que " tras cualquier actividad evaluativa es imprescindible un proceso de análisis y retro - información entre estudiantes y profesores para aprovechar y optimizar la información que la evaluación nos proporciona para el desarrollo futuro de la enseñanza y el aprendizaje... los profesores no podemos olvidar que los errores deben ser corregidos antes que se conviertan en sistemáticos, por ello, la retro - 
información debe tener lugar en un tiempo cercano a la actividad de aprendizaje o la evaluación y es necesario que cumpla algunas condiciones " (Escudero, 2010, p.20). La retroalimentación colaborativa como: retroalimentación colaborativa entre alumnos y profesores y la información retroactiva (feedback) sobre el aprendizaje.

Blázquez (2008) en el texto evaluar en Educación Física, enumera algunas formas graduales de implicación para la participación del alumnado en su propia evaluación:

- El alumno anota, en una ficha a su disposición, la valoración que él ha obtenido.

- El alumno valora su trabajo en relación con el resultado previsto.

- Suponiendo que el profesor haya dado normas claras sobre la realización, el alumno da su criterio valorativo sobre su propio trabajo. (p.57).

Inicialmente me parece interesante la propuesta de vincular progresivamente al estudiante en su propio proceso de evaluación, ya que se requieren algunas actividades eminentemente instrumentales, lo importante es que estas actividades se desarrollen en el marco de una evaluación formativa para no quedarnos en una seudo participación o participación mecánica, deben ir acompañadas de una reflexión sistemática acorde a la edad de los estudiantes.

Cambio en la función docente

La labor docente se ha transformado. Para Santos Guerra (1999)
La autoevaluación de los alumnos es una práctica poco generalizada en la universidad (y en la escuela). Es el profesor quien decide todo lo relativo a tiempos, tipos de pruebas, condiciones, frecuencia, criterios de valor.... Frecuentemente, estas decisiones no solo no son compartidas con los alumnos; sino que no son conocidas por ellos (p.384).

A nivel escolar, durante mucho tiempo, ha sido impuesta y/o propuesta exclusivamente por el profesor que determina ¿qué se evalúa?, ¿cómo se evalúa? y cuándo se evalúa? Es necesario transitar a un profesor que desarrolla acciones de acompañamiento y tutoría.

La autoevaluación fundamentada en una perspectiva de dignidad humana y democracia es propuesta por Fernández Balboa (2005) ya no solo como acto sino también como proceso y espacio para el desarrollo personal y social. Transformar la autoevaluación no es fácil ni es una tarea con resultados automáticos, requiere tiempo y esfuerzo especialmente en su sustento ético y humanista; el rol y la función del profesor de control, orden y autoridad epistemológica y deontológica debe ser revisada y ajustada en la práctica escolar cotidiana.

\section{Instrumentos para la autoevaluación en la Educación Física escolar}

Esta es la parte más difícil de implementar en la autoevaluación de los estudiantes, es llevar del dicho al hecho los desarrollos, es decir como los planteamientos teóricos se vuelven acciones prácticas en la clase de Educación Física a partir de los principios y recomendaciones para una evaluación formativa, como es utilizar una diversidad 
de técnicas e instrumentos o herramientas de evaluación complementarios.

Por ejemplo Blázquez (2008) enumera algunos instrumentos, unos exclusivos para la autoevaluación de los estudiantes y otros que pueden adoptarse para dar mayor protagonismo al estudiante como el cuaderno de evaluación del estudiante, las fichas individuales de evaluación y la ficha de autoevaluación la cual debe ser diligenciada exclusivamente por el estudiante, "es de gran utilidad para habituar al alumno a autocontrolarse y regular su propia actividad física. El momento más adecuado es la enseñanza secundaria" (Blázquez, 2008, p. 185).

Por otro lado, Velázquez y Martínez (2004) nos cuentan una experiencia para la elaboración y puesta en práctica del plan de acondicionamiento físico que denominaron memoria de evaluación del plan de acondicionamiento físico, elaborada por grupos compuestos del plan inicial de acondicionamiento físico, un diario de sesiones, una reelaboración del plan inicial y un documento que recoge una reflexión de cada estudiante sobre el proceso de elaboración y aplicación del plan y su opinión sobre la orientación didáctica dada a los contenidos de condición física. En este caso en algunas de las fases los estudiantes desarrollan actividades de autoevaluación.

López, Barba, González (2006) presentan el auto informe y la entrevista personal como un posible instrumento de autoevaluación de los estudiantes:

La redacción de autoevaluación busca, por una parte, una reflexión personal por parte del alumnado y el posibilitar un diálogo posterior con él; y por otra, el reflejo y la transformación de la evaluación continúa en evaluación final al terminar cada trimestre. Lo hacemos así porque queremos que la evaluación final y sumativa (y la obligada calificación) tengan sobre todo un sentido y una finalidad formativa (p. 5).

En cuanto a las entrevistas, se ubican "como mecanismo de regulación, diálogo y búsqueda de acuerdo cuando las autoevaluaciones y autocalificaciones son muy divergentes con nuestra valoración (del profesor) (López, Barba, Gonzales, 2006, p.5). En el marco de una evaluación formativa varios autores presentan una serie de recomendación para desarrollar una buena autoevaluación en el aula, como la entrega de criterios de evaluación (Panadero y Alonso - Tapia, 2013).

Los ejemplos y experiencias anteriores muestran como las diferentes técnicas e instrumentos de evaluación pueden y deben ajustarse a las condiciones de los estudiantes en la autoevaluación. Para no hacer un proceso incompleto es necesaria una aproximación previa con un conocimiento básico de los fundamentos y perspectivas de la autoevaluación como un proceso formativo, adaptadas al estudiante.

Eso sí no podemos despertar falsas expectativas y creer que de un día para otro se cambia la evaluación y mucho menos la autoevaluación de los estudiantes porque son procesos que requiere tiempo y actividades para superar los modelos centrados en la racionalidad técnica o instrumental que buscan un rendimiento medible solamente. 


\section{Al evaluar y autoevaluarse también se aprende.}

Al evaluar también se aprende, y por consiguiente, también es susceptible de poder desarrollar actividades y acciones concretas para aprender a autoevaluarse, se aprende, autoevaluándose y aprendiendo de los errores. "el primer movimiento de una estrategia educativa es provocar y ayudar a que los individuos entiendan y deseen los procesos de evaluación como valiosas oportunidades para el aprendizaje y la autodeterminación” (Pérez, 2012, p.240).

Porque aprender a autoevaluarse es una meta a largo plazo que requiere "fragmentar progresivamente" la profunda cultura construida desde las prácticas tradicionales, las creencias e imaginarios perpetuados desde hace muchos años para lograr cambios en la autoevaluación y convertirla en una actividad frecuente en el aula, "por ello es necesario desarrollar métodos pedagógicos orientados a desarrollar la autoevaluación" (Castillo y Cabrerizo, 2009, p.221) con referentes, principios y procedimientos de autoevaluación como una estrategia para la valoración del trabajo académico de los estudiantes.

Se requiere implementar en la institución educativa una política con acciones concretas para que el profesor desde los primeros grados brinde la posibilidad de desarrollarla y asumirla como una práctica rutinaria y necesaria y él a su vez la aplique respecto a su desempeño docente. Winne y Hadwin (1998) citado por (Panadero y Alonso- Tapia, 2013, p.556) expresan que "fomentarla (la autoevaluación de los estudiantes) implica entrenar tal proceso de forma explícita, intentado garantizar que los alumnos aprendan a autoevaluarse, es decir potenciar toda una serie de procesos metacognitivos - planificación, supervisión y evaluación".

Casanova (2010) respecto a la formación en autoevaluación reitera que:

...hay que considerar la
conveniencia, primeramente, de
introducir su práctica de modo
habitual. Con diferentes grados de
complejidad, según las edades, el
alumnado es capaz de valorar su
propia labor y el grlado de
satisfacción que le produce.
Simplemente hay que darles pautas
para que lo haga con seriedad y con
corrección - arbitrariamente ni por
juego - y que sepa la influencia que
su juicio va a tener en la valoración
global que se realice posteriormente
sobre su actuación y progresos (p.
86).

En estas condiciones, propongo que cada miembro de la comunidad educativa, especialmente los profesores y directivos docentes más que conocer y repetir unos conceptos sobre la evaluación educativa y la autoevaluación de los estudiantes comprendan los procesos e implicaciones formativas que tiene su implementación.

Un reto y tarea que tenemos los educadores es reconocer la autoevaluación como un procesó articulado a la heteroevaluación y a la coevaluación, no es un esquema regido y prediseñado. Educar desde la autoevaluación permite el ejercicio de la participación del estudiante, nuevas relaciones en el aula y otras formas de entender el conocimiento.

Previo esto necesitamos construir un marco con referentes teóricos sólidos y definir técnicas, instrumentos y 
procedimientos operativos específicos pero adaptables para la Educación Física escolar en los diferentes contextos y grados, a partir de las propuestas, experiencias e investigaciones que sobre el tema se desarrollen en el país.

\section{Conclusiones}

La tarea primordial en el ámbito institucional y de las áreas es convertir la autoevaluación de los estudiantes en una pieza fundamental en los procesos de evaluación que responda y se aplique de acuerdo al contexto pero "no se trata de declarar la autoevaluación única forma de evaluación, sino sacar partido de ella como medio de evaluación y del desarrollo del criterio propio" (Blázquez, 2008, p. 56). Los procesos de autoevaluación conllevan un aprendizaje tanto para los docentes como para estudiantes.

Por ello, en cada institución educativa se requiere una política con procesos claros y precisos con relación a los diferentes ámbitos de la evaluación escolar en el plan de desarrollo institucional, los planes de mejora y el PEI. Por ejemplo, en los sistemas institucionales de evaluación de los estudiantes que orientan y regulan la evaluación de los estudiantes en la educación básica y media, más que estar como un numeral o ítems del documento, es necesario definir puntualmente sus características y procedimientos para que no se pierda en un mar de actividades. Para cerrar, me aunó a las palabras de Pérez (2015):

...se necesita un currículo y una pedagogía que ayude a cada individuo a construirse de manera singular y creativa". Además de "más evaluación educativa, más ayuda, más orientación, más apoyo y tutorización cercana y menos calificación fría, externa, distante y selectiva en la enseñanza obligatoria (p.73).

Pero ante todo necesitamos que más allá de las buenas intenciones y palabras bonitas en la autoevaluación de los estudiantes en la Educación Física escolar se trabaje para comprometer e implicar al estudiante con autodeterminación en su aprendizaje y su formación personal y académica. Resignificar y reconstruir los procesos de autoevaluación solo se logra en la medida que cada uno de nosotros desarrollemos acciones para tal fin y en este sentido tenemos mucho por hacer.

\section{Referencias}

Álvarez Méndez, J. M. (2009). Evaluar el aprendizaje en una enseñanza centrada en competencias. En Gimeno Sacristán J. Educar por competencias ¿Qué hay de nuevo? Madrid, Morata.

Blázquez, D. (2008). Evaluar en Educación Física. $10^{\circ}$ edición. Inde Publicaciones. Barcelona. p. 57, p.185.

Casanova (2010). Manual de evaluación educativa. Muralla, Madrid.

Camacho H, Castillo E., Monje J. (2015). Educación Física Programas de $6^{\circ} \mathrm{A}$ $11^{\circ}$.Segunda edición. Editorial Kinesis. Armenia. p. 50.

Castillo S. Cabrerizo J. (2010). Evaluación educativa de aprendizaje y competencias. Pearson Educación, Madrid.

Díaz, J. (2005). El curriculum de la Educación Física en la reforma educativa. Tercera edición. Inde Publicaciones. Barcelona. 
Escudero T. (2010). Sin tópicos ni malentendidos: fundamentos y pautas para una práctica evaluadora de calidad en la enseñanza Universitaria. Documento 09. 2010. Instituto de Ciencias de la Educación. Universidad de Zaragoza.

Filip Dochy, Mien Segers y Sabine Dierick (2002). Nuevas Vías de Aprendizaje y Enseñanza y sus Consecuencias: una Nueva Era de Evaluación. Revista Docencia Universitaria Vol. 2 N. 2 Ediciones U de Murcia.

García Carrillo L.S. (2016). La importancia de la formación docente en Evaluación Revista Educación y Cultura. Mayo de 2016. Edición $N^{\circ}$ 114. Centro de Estudios e investigaciones Docentes. CIED FECODE. Bogotá.

García Carrillo L.S. (2012). Principios para una evaluación formativa en la Educación Física escolar. En Revista Kinesis $\mathrm{N}^{\circ} 59$. Editorial Kinesis Armenia.

Guio F (2012). Educación Física: tendencias y prácticas en evaluación de los aprendizajes en colegios distritales de Usaquén. Revista Educación Física y Deporte. N 31- 2, 863 - 870, 2012, Funámbulos editores. Universidad de Antioquia. Medellín.

Fernández - Balboa J. M. (2005). La auto - evaluación como práctica promotora de la democracia y dignidad. Sicilia y Fernández -Balboa (coordinadores) La otra cara de la enseñanza. La Educación Física desde una perspectiva crítica. Inde publicaciones, Barcelona.
Fraile A (Coord.) (2004). Didáctica de la Educación Física. Una perspectiva crítica y transversal. Editorial Biblioteca nueva. Madrid.

López V., Barba J.J., Gonzales M, (2006). ¿Debe el alumno participar en la evaluación? Propuestas y experiencias en primaria. Bloc Concejo Educativo de Castilla y León. Movimiento de renovación pedagógica. www.concejoeducativo.org consultado en enero de 2016.

MEN (2000). Lineamientos curriculares Educación Física, Recreación y Deporte áreas obligatorias $\mathrm{y}$ fundamentales.

MEN (2010). Orientaciones pedagógicas para la Educación Física, Recreación y Deporte. Documento 15.

Moreno T. (2011) Frankestein evaluador. Revista de la Educación Superior. Vol. 40, núm. 160. México, octubre diciembre/2011.

Murillo F. J. y Hidalgo N. (2015) Dime Cómo evalúas y Te Diré Que Sociedad Construyes. Editorial Revista Iberoamericana de Evaluación Educativa, 2015, 8 (1), 5-9.

Panadero E. y Alonso - Tapia J. (2013). Autoevaluación: Connotaciones teóricas y prácticas. Cuando ocurre, Como se adquiere y que hacer para Potenciarla en nuestro Alumnado. Electronic Journal of Research in Educational Psychology, SeptiembreSin mes, 551-576.

Pérez A. (2015). Aprender a pensar para poder elegir. La urgencia de una nueva 
pedagogía. En Gimeno J. (comp.) (2015) Los contenidos. Una reflexión necesaria.Ediciones Morata Madrid.

Pérez A. (2012). Educarse en la era digital. La escuela educativa. Ediciones Morata Madrid.

Perrenoud P. (2008). La evaluación de los alumnos. De la producción de la excelencia a la regulación de los aprendizajes. Entre dos lógicas. Editorial Colihue. Alternativa Pedagógica Didáctica. Buenos Aires.

Popham, J. (2013). La evaluación trans formativa. El poder transformador de la evaluación educativa. Nacea. Madrid.

Román M (2011). Autoevaluación: estrategia y componente esencial para el cambio y la mejora escolar. Revista Iberoamericana de Educación N55. P107 a 136.

Santos Guerra M. A. (2010). La evaluación como aprendizaje. Una flecha en la diana. Tercera edición. Bonum.

Santos Guerra, M. A. (1996). La evaluación un proceso de dialogo, compresión y mejora. Málaga. Ediciones Aljibe.

Velázquez R y Martínez M. A. (2004). la autoevaluación y la coevaluación en el aprendizaje en el aprendizaje autónomo. En Hernández J. L., Velázquez R. (coord.) La evaluación en Educación Física. Investigación y práctica en el ámbito escolar. Grao. Barcelona. 


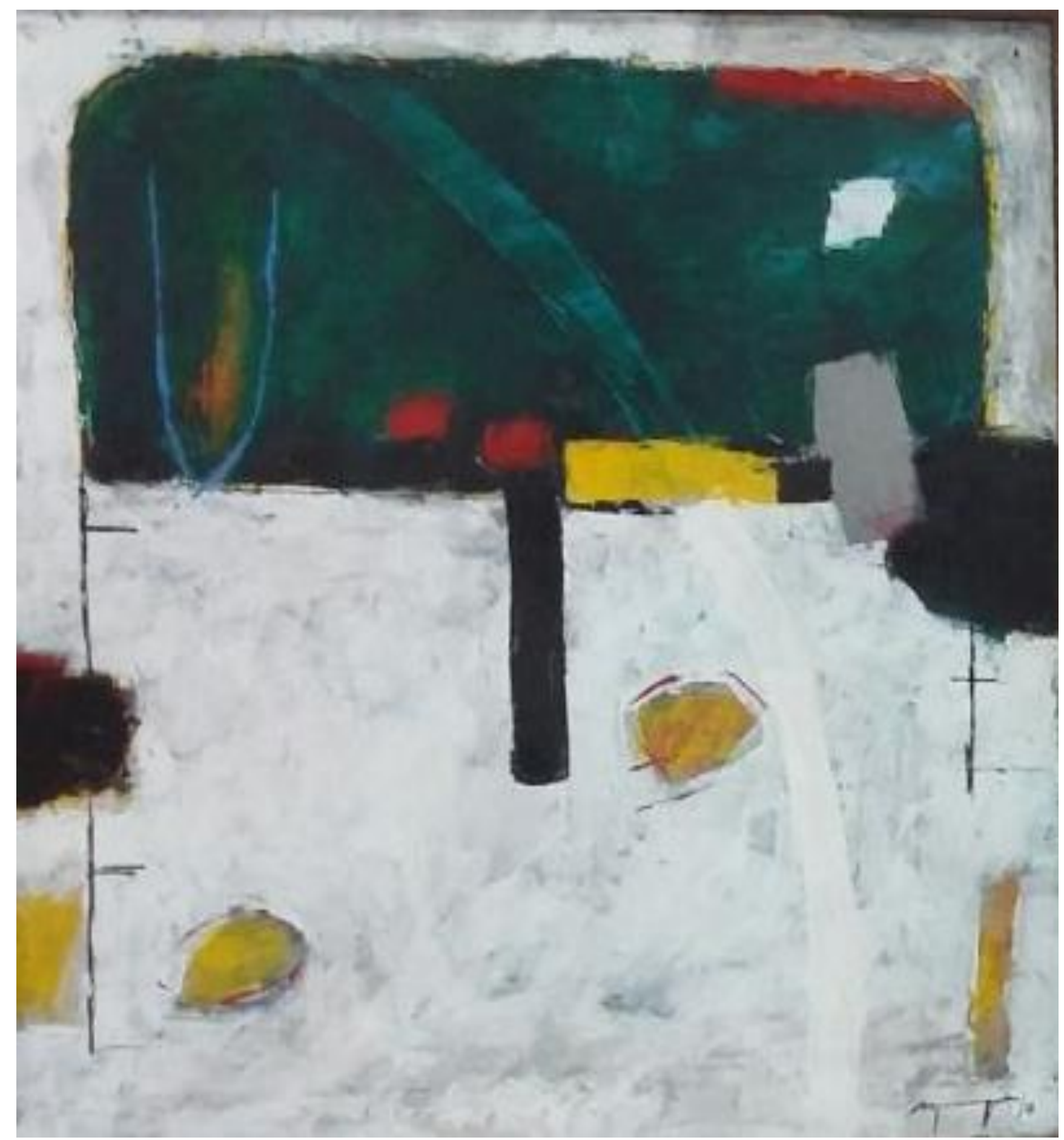

Título de la obra: de la serie selva

Técnica: pigmentos industriales sobre polietileno

Dimensiones de la obra: 100 c.ms. X 100 c.ms.

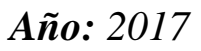

Nombre de la imagen adjunta: 001 